\title{
Directional asymmetries reveal a universal bias in adult vowel perception
}

Matthew Masapollo, Linda Polka, Monika Molnar, and Lucie Ménard

Citation: The Journal of the Acoustical Society of America 141, 2857 (2017); doi: 10.1121/1.4981006

View online: https://doi.org/10.1121/1.4981006

View Table of Contents: http://asa.scitation.org/toc/jas/141/4

Published by the Acoustical Society of America

\section{Articles you may be interested in}

Modulation of auditory-motor learning in response to formant perturbation as a function of delayed auditory feedback

The Journal of the Acoustical Society of America 141, 2758 (2017); 10.1121/1.4981139

Sensorimotor adaptation affects perceptual compensation for coarticulation

The Journal of the Acoustical Society of America 141, 2693 (2017); 10.1121/1.4979791

Adaptation to novel foreign-accented speech and retention of benefit following training: Influence of aging and hearing loss

The Journal of the Acoustical Society of America 141, 2800 (2017); 10.1121/1.4980063

Analysis of human scream and its impact on text-independent speaker verification

The Journal of the Acoustical Society of America 141, 2957 (2017); 10.1121/1.4979337

Infants adapt to speaking rate differences in word segmentation

The Journal of the Acoustical Society of America 141, 2569 (2017); 10.1121/1.4979704

Intra-speaker and inter-speaker variability in speech sound pressure level across repeated readings

The Journal of the Acoustical Society of America 141, 2353 (2017); 10.1121/1.4979115 


\title{
Directional asymmetries reveal a universal bias in adult vowel perception $^{\text {a) }}$
}

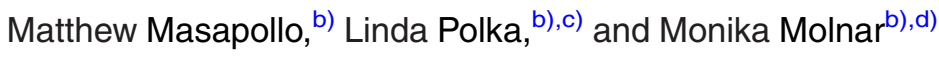 \\ School of Communication Sciences and Disorders, McGill University, 2001 McGill College, 8th Floor, \\ Montreal, Quebec, H3A 1G1, Canada \\ Lucie Ménard ${ }^{\mathrm{b}}$ ) \\ Département de Linguistique, Université du Québec à Montréal, Pavillon J.-A. De sève, DS-4425, 320, \\ Sainte-Catherine Est, Montréal, Quebec, H2X 1L7, Canada
}

(Received 24 October 2016; revised 29 March 2017; accepted 4 April 2017; published online 21 April 2017)

\begin{abstract}
Research on cross-language vowel perception in both infants and adults has shown that for many vowel contrasts, discrimination is easier when the same pair of vowels is presented in one direction compared to the reverse direction. According to one account, these directional asymmetries reflect a universal bias favoring "focal" vowels (i.e., vowels whose adjacent formants are close in frequency, which concentrates acoustic energy into a narrower spectral region). An alternative, but not mutually exclusive, account is that such effects reflect an experience-dependent bias favoring prototypical instances of native-language vowel categories. To disentangle the effects of focalization and prototypicality, the authors first identified a certain location in phonetic space where vowels were consistently categorized as / $\mathrm{u} /$ by both Canadian-English and Canadian-French listeners, but that nevertheless varied in their stimulus goodness (i.e., the best Canadian-French $/ \mathrm{u} /$ exemplars were more focal compared to the best Canadian-English $/ \mathrm{u} /$ exemplars). In subsequent AX discrimination tests, both Canadian-English and Canadian-French listeners performed better at discriminating changes from less to more focal /u/'s compared to the reverse, regardless of variation in prototypicality. These findings demonstrate a universal bias favoring vowels with greater formant convergence that operates independently of biases related to language-specific prototype categorization. (C) 2017 Acoustical Society of America.
\end{abstract}

[http://dx.doi.org/10.1121/1.4981006]

[JFL]

Pages: $2857-2869$

\section{INTRODUCTION}

Many aspects of human cognition are shaped by experience, while others appear to be governed by universal principles. A specific example comes from speech perception, where the fundamental perceptual operation is the mapping of an input acoustic signal onto phonological units. Considerable evidence, spanning some 60 years of research, indicates that this mapping process is influenced by both the intrinsic acoustic-phonetic properties of speech sounds and the structure of the language-specific phoneme inventory [see Cutler (2012) for a review]. The current work examines the nature of this complex interplay between universal and experiential factors in the context of an important phenomenon in speech perception: directional asymmetries in vowel discrimination.

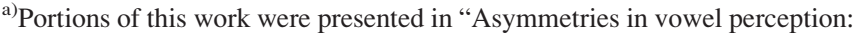
Effects of formant convergence and category goodness," in Proceedings of the 18th International Congress of Phonetic Sciences, Glasgow, Scotland, United Kingdom (August 2015).

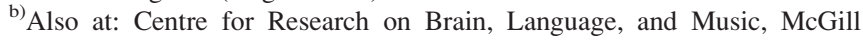
University, 3640 de la Montagne, Montreal, Quebec H3G 2A8, Canada.

${ }^{c}$ Also at: Centre for Research on Brain, Language, and Music, McGill University, 3640 de la Montagne, Montreal, Quebec H3G 2A8, Canada. Electronic mail: linda.polka@mcgill.ca

${ }^{d)}$ Currently at the Basque Center on Cognition, Brain and Language. Donostia, Spain.
}

Research on cross-language vowel perception in both infants and adults has shown that for many betweencategory vowel contrasts, discrimination is easier when the same pair of vowels is presented in one direction compared to the reverse direction (see Polka and Bohn, 2003, 2011, for reviews). For example, Polka and colleagues found that both German- and English-learning infants performed better at discriminating the change from German $/ \mathrm{y} /$ to $/ \mathrm{u} /$, compared to the reverse change from $/ \mathrm{u} /$ to $/ \mathrm{y} /$ (Polka and Werker, 1994; Polka and Bohn, 1996). These directional asymmetries have been reported in numerous infant vowel discrimination studies using a wide range of vowel contrasts from across phonetic space in several behavioral paradigms (i.e., habituation, operant conditioning). Figure 1(a) shows some of the vowel contrasts that have been examined in infant vowel discrimination studies; each contrasting vowel pair is connected with an arrow indicating the direction of change that was easier to discriminate.

Critically, these asymmetries cannot be explained by reference to native vowel inventories because they emerge in infants' discrimination of both native and non-native (foreign language) vowel contrasts. These effects are also not consistent with a bias related to simple acoustic dimensions, such as pitch, amplitude, or duration, as these variables were wellcontrolled in the test stimuli used across studies. However, these asymmetries in general, with few exceptions, could be 
predicted by considering the relative position of each vowel within articulatory/acoustic vowel space (defined by $F 1-F 2$ ). More precisely, infants tend to perform better at discriminating a change from a relatively less to a relatively more peripheral vowel, compared to the same change presented in the reverse direction. Although these findings were initially interpreted as a peripheral vowel bias, as explained below, with further research it became clear that this bias is related to formant convergence patterns that involve more than just $F 1$ and $F 2$.

Polka and Bohn (2003) initially proposed that this early vowel bias plays an important role in the development of vowel perception by establishing stable referents that help young infants attend to and differentiate vowels during the period when they are learning phonetic categories. Broadly consistent with this idea, more recent studies have shown that linguistic experience fine-tunes this initial vowel bias to optimize access to native-language vowel categories during speech processing (Polka and Werker, 1994; Polka and Bohn, 2011; Pons et al., 2012; Dufour et al., 2013; Tyler et al., 2014). For example, with respect to German /u-/y/, monolingual English-speaking adults continue to show the same asymmetry as English- and German-learning infants, while German-speaking adults show symmetric (and near perfect) discrimination of this contrast (Polka and Bohn, 2011). A similar pattern of developmental change emerged when Danish-speaking adults and Danish-learning infants were tested on a native contrast (i.e., Danish /e/-/ø/) and a non-native contrast (i.e., British English / $/$ /-/u; Polka and Bohn, 2011), and when Spanish- and Catalan-learning infants were tested on discrimination of Catalan /i-e/ (Pons et al., 2012). While a developmental shift from a universal to a language-specific pattern of vowel perception is expected for non-native contrasts, it is important to note that asymmetries in adults' perception of some native contrasts have also been observed (Repp et al., 1979; Cowan and Morse, 1986; Repp and Crowder, 1990).

On the basis of these findings, Polka and Bohn (2011) more recently formulated the Natural Referent Vowel (NRV) Framework. According to this framework, young infants from across cultures come to the task of language acquisition universally biased toward certain vowels which act as natural reference points (or perceptual attractors) within phonetic space. As infants accrue experience listening to a specific language, the perceptual vowel space is finetuned to align with the regularities of the native-language vowel system (Polka and Werker, 1994; Polka and Bohn, 2011). Nevertheless, this initial bias will continue to operate

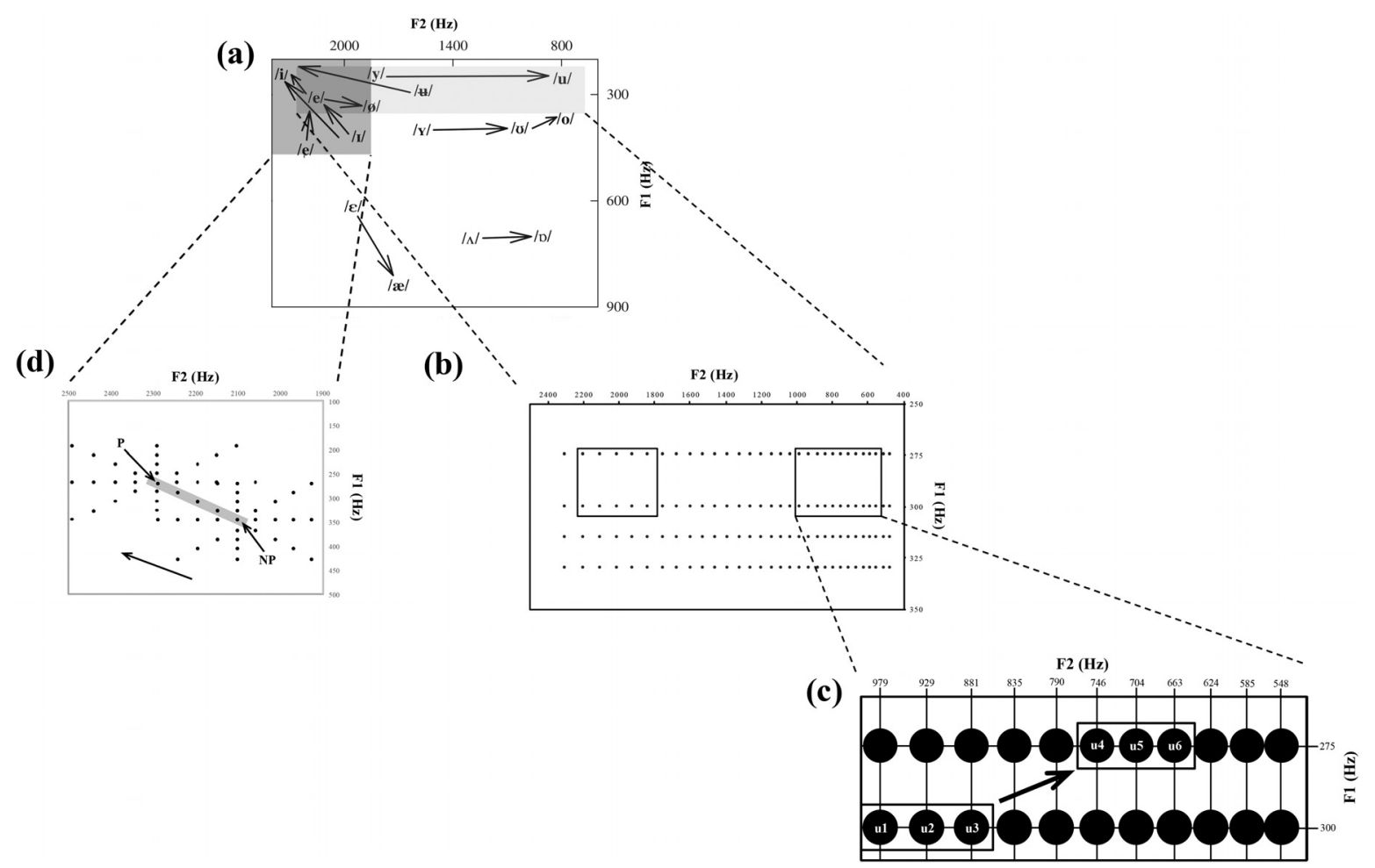

FIG. 1. (a) Schematic illustration of articulatory-acoustic vowel space based on the first two formants. Vowel contrasts showing asymmetries in infant vowel perception are plotted; arrows point in the direction that is easier to discriminate. The light gray area highlighted in this space corresponds to the region covered by the four vowel series synthesized for use in the present study. The dark gray area highlighted in this space corresponds to the region covered by the vowel series synthesized for use in Kuhl (1991). (b) Magnified view of the vowel stimuli presented to English- and French-speaking listeners for identification and goodness ratings (experiment 1). The formant frequencies were equally spaced on a psychophysical basis (on the bark scale; Zwicker and Terhardt, 1980). Embedded within this space are the tokens that were consistently identified as exemplars of English $/ \mathrm{i} /$ or French $/ \mathrm{y} /(F 1=275$ and $300 \mathrm{~Hz} ; F 2=1753$ to $2202 \mathrm{~Hz})$ and as exemplars of English /u/ or French /u/ ( $F 1=275$ and $300 \mathrm{~Hz} ; F 2=548$ to $979 \mathrm{~Hz}$; outlined in black). (c) The six /u/ tokens used in the discrimination task (experiment 2) are outlined in black, and labeled $u_{1}$ thru $u_{6}$. Stimuli $u_{1}, u_{2}$, and $u_{3}$ formed the less focal vowel set, and stimuli $u_{4}, u_{5}$, and $u_{6}$ formed the more focal set. The arrow points in the direction that NRV predicts will be easier to discriminate (see text for explanation). (d) The formant frequency values (in Hz) for the /i/ vowel stimuli used in Kuhl (1991). P is the "prototype" vowel and NP is the "non-prototype" as specified by Kuhl (1991). The gray area highlighted in this space corresponds to vowel stimuli on a common vector shared between P and NP. Kuhl (1991) reported directional asymmetries in infant discrimination of the stimuli along this common vector; the arrow points in the direction that was easier for infants to discriminate. 
in adult language users, emerging most clearly in the perception of non-native vowel contrasts.

While recent progress has been made in interpreting asymmetries, we are still left with the question as to why perceivers are universally biased toward some vowels over others. As mentioned above, Polka and Bohn's (2003) initial hypothesis was that this bias favored vowels in the periphery of phonetic space. However, several asymmetries that were not predicted by this view revealed that the simple description of vowels within $F 1 / F 2$ acoustic space was not adequate. Instead, researchers needed to consider additional formants (not just $F 1$ and $F 2$ ) and also the spectral proximity of formants to each other. NRV directly addresses this issue by postulating that asymmetries reflect a universal perceptual sensitivity to formant proximity (Polka and Bohn, 2011; see also Schwartz et al., 2005). The fundamental idea is that extreme vocalic articulations give rise to acoustic signals with well-defined spectral prominences due to formant frequency convergence, or "focalization"; such articulations in turn lead to acoustic signals that exhibit increased spectral salience and acoustic-perceptual stability. To clarify, a good deal of research has shown that when spectrally adjacent formants move close together in frequency there is a mutual reinforcement of their acoustic energy, such that the amplitude of each formant increases. As a result, when formants converge acoustic energy becomes focused into a narrower spectral region [see Stevens (1989) and Kent and Read (2002) for a discussion]. Critically, a maximal degree of formant convergence is observed for vowels found at the periphery of phonetic space, which also have the most extreme vocal tract postures. For example, $F 2$ and $F 3$ and $F 4$ converge (i.e., are close in frequency) for /i/ (which is the highest front vowel), and $F 1$ and $F 2$ are spectrally close to each other for /a/ (which is the lowest back vowel) as well as $/ \mathrm{u} /$ (which is the highest back vowel). Because the corner vowels $(/ \mathrm{i} / / \mathrm{u} / \mathrm{a} /)$ exhibit a maximal degree of formant convergence, they have been referred to as "focal vowels" in the speech literature (Schwartz et al., 1997). ${ }^{1}$ Thus, the hypothesis is that more focal vowels have a privileged perceptual status due to their well-defined spectral prominences (i.e., they are easier to detect and encode in memory compared to vowels with less well-defined spectral prominences). For many, but not all, vowel contrasts a relatively more versus a relatively less peripheral location in the standard $F 1 / F 2$ space aligns with differences in focalization. However, it is important to note that focalization and peripherality are conceptually not the same. Focalization takes into accounts the first three to four formants and their spectral position relative to each other, not just the vowel position within a simple $F 1 / F 2$ space.

Indeed, there is some experimental evidence consistent with the claim that discrimination asymmetries are driven by focalization. Schwartz and Escudier (1989) tested French adult listeners on their ability to discriminate exemplars of French /e/ synthesized with the same $F 1, F 2$, and $F 4$ values, but with different $F 3$ values. Although all tokens were perceived as /e/, one vowel variant had an $F 3$ frequency that was an equal psychophysical distance between $F 2$ and $F 4$, another had an $F 3$ spectrally closer to $F 2$ and yet another had an F3 closer to F4. Thus, these /e/ vowel tokens systematically differed in their degree of formant proximity. French adults showed directional asymmetries consistent with an effect of focalization. Specifically, discrimination was better when they heard a less focal /e/ followed by a more focal /e/, compared to when the same vowels were presented in the reverse order (i.e., more focal /e/ followed by less focal /e/). Although asymmetric patterns were reported in this study, these effects were interpreted as focalization effects only later when similar asymmetries emerged in infant vowel perception research (Schwartz et al., 2005).

There are several reasons, however, to suggest that language experience could have played a role in shaping the perceptual asymmetry observed by Schwartz and Escudier (1989). In a seminal study by Kuhl (1991), it was reported that language experience affects listeners' perception of vowel stimuli from within a given phonetic category. She presented English adult listeners with a range of synthetic /i/ vowels that systematically varied in their first $(F 1)$ and second formants (F2). Listeners consistently perceived the stimuli in a particular part of vowel space as better exemplars of /i/, indicating that the category has a graded, internal structure. Interestingly, the stimuli perceived as prototypic category members also matched the average acoustic production values of /i/ (Peterson and Barney, 1952), suggesting that there may be a close correspondence between the mean stimulus values experienced in the input signal and the stored prototype [although this outcome conflicts with other studies, which show that prototypicality judgments are often more peripheral compared to the average production values reported in a corpus distribution (see, e.g., Johnson et al., 1993; Lively and Pisoni, 1997; Diesch et al., 1999; Whalen et al., 2004)].

On the basis of these findings, Kuhl (1991) identified a good exemplar as the prototype, and a poor exemplar as the non-prototype. She then synthesized 32 category variants that orbited the prototype and non-prototype in equal psychophysical steps [Fig. 1(d) presents a F1/F2 plot of Kuhl's (1991) /i/ stimulus array]. Using a change detection paradigm, she found that both English-speaking adults and English-learning infants (at 6-months of age) showed greater discrimination of the non-prototype from its variants, compared to discrimination of the prototype from its variants. In sharp contrast, rhesus macaques were found to discriminate the prototype and non-prototype variants of /i/ to the same degree. Kuhl (1991) reasoned that it might have been harder for the human adult and infant listeners to discriminate the prototype from its variants, than the non-prototype from its variants, because they (unlike the macaques) were organizing the stimuli into a category.

A second finding of the study by Kuhl (1991), reinforcing the interpretation that human vowel perception is influenced by category learning, was obtained in the discrimination task with infants. A subset of the /i/ stimuli were shared between the orbitals surrounding the prototype and non-prototype. An analysis of infants' discrimination of these stimuli revealed a directional asymmetry, such that they performed better at discriminating the change going in the direction from the non- 
prototypic to the prototypic /i/, compared to the same change presented in the reverse direction.

On the basis of these and other compatible findings, Kuhl (1993) proposed the Native Language Magnet (NLM) Theory, which argues that category learning influences perceptual patterns, such that listeners become biased toward native prototypes. The basic idea is that language-experience gives rise to prototypes that are perceptual "hot spots" that act like "perceptual magnets" and warp the perceptual space around the prototype. This in turn facilitates access to native vowel categories by increasing internal category cohesion, and maximizing the perceptual distance between category edges (see also Feldman et al., 2009). The prototype's magnetic properties can be demonstrated experimentally in two ways: (1) As increased generalization (reduced discrimination) for vocalic exemplars close to the prototype in psychophysical space; and (2) as a directional asymmetry in the discrimination of prototypic and non-prototypic exemplars that fall within a given vowel category; namely, poorer discrimination of a prototypic to non-prototypic change compared to a non-prototypic to prototypic change.

There are several reasons, however, to suspect that the asymmetry reported by Kuhl (1991) reflects something besides the proposed prototype magnet effect. The first concerns a methodological criticism of her study. Specifically, it has been argued that listeners might have shown enhanced discrimination of the non-prototype and its variants if the non-prototype was across a category boundary and was not consistently identified as /i/ (see, e.g., Lotto et al., 1998). Since the listeners in Kuhl's study were not explicitly asked to categorize the vowel stimuli, it was not certain whether the non-prototype was consistently identified as /i/. In fact, subsequent studies found that many listeners often identified the non-prototype as a different vowel (e.g., Iverson and Kuhl, 1995; Lively and Pisoni, 1997; Lotto et al., 1998, reported that many listeners identified the non-prototype as /e/), suggesting that the stimuli in Kuhl (1991) spanned different phonetic categories. This raises the possibility that the directional asymmetries reported by Kuhl (1991) may simply reflect a category boundary effect (cf. Iverson and Kuhl, 1995, 2000). A second reason to question Kuhl's (1991) interpretation is that the prototype was more focal (between $F 2$ and F3) compared to the non-prototype. Thus, as in the case of Schwartz and Escudier (1989), the prototype/nonprototype asymmetry is also equivocal. Specifically, it could be attributed to prototypicality effects, category boundary effects, or focalization effects.

Thus, there are several reasons to examine further the nature of directional asymmetries in vowel perception. According to the NRV framework (Polka and Bohn, 2011), vowel perception is shaped by both universal biases and experiential influences. Specifically, it is hypothesized that a universal perceptual bias favoring more focal vowels is present in early infancy; this bias will then be modified by linguistic experience in mature adult perceivers. Attunement to native-language phonetic categories begins in infancy (Kuhl et al., 1992; Polka and Werker, 1994), which eventually causes directional asymmetries to fade for native vowel contrasts but remain in place for non-native vowel contrasts
(Polka and Bohn, 2011; Pons et al., 2012). A central claim of the NRV framework is that asymmetries reflect a universal bias that is phonetically grounded in vowel acoustics. Critically, however, the existing data do not provide definitive evidence that adults show asymmetries rooted in perceptual sensitivity to differences in formant proximity, independent of prototype categorization processes. The NLM Theory (Kuhl, 1993; Kuhl et al., 2008), on the other hand, has largely ignored the role of universal biases, and instead proposes that asymmetries are a by-product of phonetic category learning induced by exposure to statistical distributions of vowels present in input speech.

To address this theoretical tension, we designed a study to examine whether adult listeners from different language backgrounds are indeed sensitive to differences in formant proximity while discriminating subtle acoustic differences that fall within a given vowel category, and, if so, whether that sensitivity operates independently of language-specific prototype categorization. It differs from earlier work by Schwartz and Escudier (1989) and Kuhl (1991) in teasing apart the effects of focalization and stimulus goodness on vowel discrimination, thus providing a strong cross-language test of the competing predictions of NRV and NLM.

Guided by previous experimental findings, we chose Canadian-English and Canadian-French as languages expected to differ in their prototype structure of the vowel /u/. Escudero and Polka's (2003) study of vowel production in Canadian-English and Canadian-French measured the spectral properties of five analogous vowel categories (/i, y, $\mathrm{u}, \varepsilon, æ /)$ produced by six native speakers in each of the two languages. They found that Canadian-French speakers consistently produced more extreme /u/ gestures (resulting in lower $F 1$ and $F 2$ values) than Canadian-English speakers, with the mean location of Canadian-French $/ \mathrm{u} /$ being more peripheral than that of Canadian-English $/ \mathrm{u} / \mathrm{in}$ phonetic space (see also Martin, 2002; MacLeod et al., 2009; Noiray et al., 2011). Thus, the acoustic structure of CanadianFrench $/ \mathrm{u} /$ is more focal (between $F 1$ and $F 2$ ) than that of Canadian-English /u/. Given these quantitative languagespecific differences in the acoustic structure of $/ \mathrm{u} /$, we hypothesized that we might also observe language-specific differences in the prototype structure of $/ \mathrm{u} /$, such that more focal variants will be more representative of the CanadianFrench /u/ category. Recall that Kuhl (1991) found a close correspondence between the typicality of /i/ exemplars and the acoustics of average production [based on /i/ production estimates reported in Peterson and Barney (1952); cf. Lively and Pisoni, 1997; Diesch et al., 1999].

The present study investigated whether directional asymmetries can be ascribed to (universal) vowel focality effects and/or to (language-specific) prototypicality effects. Experiment 1 was a perceptual study designed to determine the detailed nature of the internal structure of the $/ \mathrm{u} /$ category in Canadian-English and Canadian-French. This first experiment allowed us to determine whether we could identify the critical stimulus items for testing the competing predictions of NRV and NLM. Specifically, it was required that we obtain variants of $/ \mathrm{u} /$ that systematically differ in both their formant proximity (between $F 1$ and $F 2$ ) and category 
goodness ratings. Experiment 2 tested the predictions by asking Canadian-English and Canadian-French listeners to discriminate synthetic variants of $/ \mathrm{u} /$ that we had chosen on the basis of the results of experiment 1 .

\section{EXPERIMENT 1: VOWEL IDENTIFICATION AND GOODNESS RATINGS}

Previous phonetic descriptions summarized in Sec. I indicate that $/ \mathrm{u} /$ has different quantitative properties in Canadian-English and Canadian-French (Escudero and Polka, 2003; MacLeod et al., 2009; Noiray et al., 2011). However, it is unknown whether there is a convergence between production and perception measures of $/ \mathrm{u} /$ in both languages. Thus, experiment 1 had two goals. The first goal was to confirm that Canadian-English and Canadian-French listeners' phonetic category goodness judgments are indeed linked to differences in formant convergence patterns between $F 1$ and $F 2$. The second goal was to identify /u/ vowel stimuli that could be used to disentangle the effects of formant convergence and category goodness on asymmetries in adult vowel discrimination.

These two goals were accomplished by synthesizing an array of vowel stimuli that spanned the high/back region of the vowel space to include prototypical instances of Canadian-English $/ \mathrm{u} /$ and of Canadian-French $/ \mathrm{u} /$ that also systemically differed in the proximity between $F 1$ and $F 2$ (with the frequency distance between $F 1$ and $F 2$ varied in equal psychophysical steps). Native, monolingual CanadianEnglish and Canadian-French listeners then completed a categorization and goodness rating task with this array of vowels. We hypothesized that the location of stimuli perceived as the best $/ \mathrm{u} /$ exemplars would fall in different regions of phonetic space for each language group with the best instances of French $/ \mathrm{u} /$ being more focal variants compared to the best instances of English /u/.

\section{A. Materials and methods}

\section{Subjects}

Twenty-six adults were recruited in Montreal, including 13 native, monolingual Canadian-English listeners (mean age $=24$ yrs [standard deviation $(\mathrm{SD})=4.4$ ]; 5 males $)$ and 13 native, monolingual Canadian-French listeners (mean age $=25 \mathrm{yrs}[\mathrm{SD}=4.8]$; 7 males). To avoid potential dialectal variation effects (see, e.g., Frieda et al., 1999), only monolingual English listeners from Ontario ${ }^{2}$ and monolingual French listeners from Quebec were included in the study. Subjects were paid for their participation. Subjects had to meet the following inclusion criteria: (1) report no speech or hearing disorders and no prior linguistic or phonetics training, (2) raised in a monolingual home and educated in a monolingual school in their respective language, (3) no experience learning a second language before $10 \mathrm{yrs}$ of age, and (4) no experience conversing in a second language on a regular basis. Four additional subjects (two English; two French) were tested, but excluded from the analysis because their responses were highly irregular, and unlike their peers, failed to reveal a distinct region corresponding to "good" instances of their native-language /u/ category within the stimulus array (see details concerning the identification and goodness rating task below).

Subjects completed either the Canadian-English or Canadian-French version of the Language Experience and Proficiency Questionnaire (LEAP-Q; Marian et al., 2007). As part of this questionnaire, participants self-rated their speaking and listening proficiency in the language that was foreign to them (in English for the French monolinguals and in French for the English monolinguals) using a 1-10 rating scale $(1=$ very poor, $10=$ excellent $)$. The English subjects' median self-ratings of their French proficiency were 1.0 (range $=1-4$ ) for speaking and 2.0 (range $=1-4$ ) for understanding spoken French. The French subjects' median selfratings of their English proficiency were 3.0 for speaking (range $=1-4$ ) and 3.0 for understanding spoken English (range $=1-4$ ). Median tests showed that the English proficiency ratings by French adults and French proficiency ratings by the English adults were not statistically different $(p>0.05)$ for either speaking or comprehension.

\section{Stimuli}

The vowel stimuli used in experiment 1 included 34 tokens, which are highlighted with gray shading on Fig. 1(b). These items were selected from the larger array of 128 vowel tokens shown in Fig. 1(b), which consisted of four series of isolated vowels varying in $F 1$ (from 275 to $330 \mathrm{~Hz}$ ) and $F 2$ (from 476 to $2303 \mathrm{~Hz}$ ) in equal psychophysical steps on the bark scale (Zwicker and Ternhardt, 1980).

The stimuli were synthesized using the Variable Linear Articulatory Model (VLAM) (Maeda, 1979, 1990; Boё, 1999; Ménard et al., 2004), which generates high-quality acoustic vowel signals based on a current understanding of the articulatory-acoustic relations of the human vocal tract. As described in detail in Ménard et al. (2004), the VLAM model is based on a statistical analysis of 519 cineradiographic images of a French speaker (Bothorel et al., 1986). A Principal Component Analysis conducted on the midsagittal contours of the vocal tract revealed that $88 \%$ of the variance could be explained by the seven articulatory parameters. Each of these parameters is adjustable at a value in the range of $\pm 3.5 \mathrm{SD}$ of the mean values for this articulator in the cineradiographic images. The model can generate all vowels of the world's languages, without reference to the corpus used to extract the control parameters. Furthermore, in a study of perceptual categorization by French and English listeners, synthesized stimuli covering the model's maximal vowel space were generated (Ménard et al., 2004; Ménard et al., 2009). It was shown that native French vowels as well as native English vowels could be reliably identified by participants.

Critically, VLAM also successfully simulates the interaction among converging formant frequencies in an ecologically-valid manner. The stimuli were identical in all other respects: $f 0, F 3, F 4$, and $F 5$ were $120,2522,3410$, and $4159 \mathrm{~Hz}$, respectively. These tokens emulated an adult male voice. Each stimulus was 400-ms in duration, and had the same intonation contour. For English listeners, the four 
vowel continua varied perceptually from /u/ ("oo") to /i/ ("ee") as $F 2$ values increased. For French listeners, the continua varied perceptually from $/ \mathrm{u} /$ (oo) to $/ \mathrm{y} /$ (as in the French word "but") to /i/ (ee) as the $F 2$ values increased

As a preliminary step, pilot testing was conducted with the full array of 128 vowel tokens to select a smaller set of vowels that are identified only as /u/ by all English and all French listeners. Specifically, five monolingual CanadianEnglish and five monolingual Canadian-French listeners completed a phonetic identification and goodness rating task with the full array. They listened to each token three times in a randomized order and had to first decide whether the sounds they heard matched any of their native-language vowel categories by clicking on a word on a computer screen representing one of the target vowels. They then had to rate the quality (or category "goodness") of each vowel using a $1-5$ rating scale $(1=$ very poor, $5=$ very good $)$.

Based on the results of this identification and goodness rating task, we then selected the 34 vowels corresponding to the tokens highlighted in gray in Fig. 1(b); the excluded vowels were judged as a non-match or a match with low goodness rating (below 2.4 on average). This final stimulus set included 22 back vowels targeting English /u/ and French $/ \mathrm{u} /$ vowel $(F 1=275$ and $300 \mathrm{~Hz} ; F 2=548$ to $979 \mathrm{~Hz})$, and 12 front vowels targeting English /i/ and French /i/ and /y/ $(F 1=275$ and $300 \mathrm{~Hz} ; F 2=1753$ to $2202 \mathrm{~Hz})$. Note that we also selected clear instances of $/ \mathrm{i} /$ and $/ \mathrm{y} /$ and synthesized two additional filler vowels (/o/ ["oh"] and /ə/ ["uh"]) to include in the stimulus set to provide some variation in vowel quality during the perception task. This also made it easier to assess whether subjects were successful in identifying vowel quality differences using key words.

\section{Procedure and design}

Listeners were presented with randomized sequences of the 36 vowel stimuli (the 34 initial tokens [highlighted in Fig. 1(b)] and the 2 filler vowels) and were asked to identify each token in terms of their own native-language vowel categories, and then judge its category goodness using a 1-5 rating scale $(1=$ very poor, $5=$ very good). Each test session included 360 trials (10 trials for each stimulus).

The experiment was conducted in a sound-treated laboratory room with participants facing a computer screen. The stimuli were presented over headphones at a comfortable loudness level of $65 \mathrm{~dB}$ in a self-paced manner; after each vowel presentation, subjects decided themselves how much time they needed to respond. The identification and goodness rating responses were collected using Praat (Boersma and Weenink, 2016). During stimulus presentation, words containing the target vowels and a rating scale appeared on the computer screen. By choosing and clicking on one of the words, the subjects decided what sound they heard (identification). Then, using a 5-point rating scale, they judged the quality of the sounds (category goodness).

For the English-speaking subjects, the following target words appeared on the screen: "boo" (for the vowel /u/), "bee" (for the vowel /i/), "bowl" (for the vowel /o/), "bug" (for the vowel /ə/), and an " $\mathrm{X}$ " to choose if the vowel could not be matched to any of the other vowel choices. The rating scale, presented at the bottom of the screen, consisted of five boxes labeled from 1 to 5 . The scale endpoints were also labeled with "very poor exemplar (1)" and "very good exemplar (5)." For the French-speaking subjects, the following target words were presented on the screen: "boue" (for the vowel /u/), "bu" (for the vowel /y/), "bille" (for the vowel /i/), "bas" (for the vowel /a/), and "beau" (for the vowel /o/) and option $\mathrm{X}$ for no match.

\section{B. Results}

As mentioned above, in this study we were concerned only with the identification and goodness ratings of the 22 back vowels indicated in Figs. 1(b) and 1(c). Recall that subjects identified and rated each stimulus 10 times. The entire array of 22 back vowels was consistently identified as /u/ by both French and English adults; identification rate (across all subjects) was $100 \%$ for many tokens and was above $85 \%$ for all tokens. For each subject, a composite goodness score (hereafter referred to as goodness score) was computed for each vowel by adding up the ratings across every trial in which the token was categorized as $/ \mathrm{u} /$; trials in which the vowel was not identified as $/ \mathrm{u} /$ received a 0 score. Thus, goodness scores ranged from 0 (never identified as $/ \mathrm{u} /$ ) to 50 [identified as $/ \mathrm{u} /$ and assigned the highest rating (5) on all 10 trials]. The median goodness scores for each $/ \mathrm{u} /$ token are plotted in Fig. 2 with the results from the English adults on

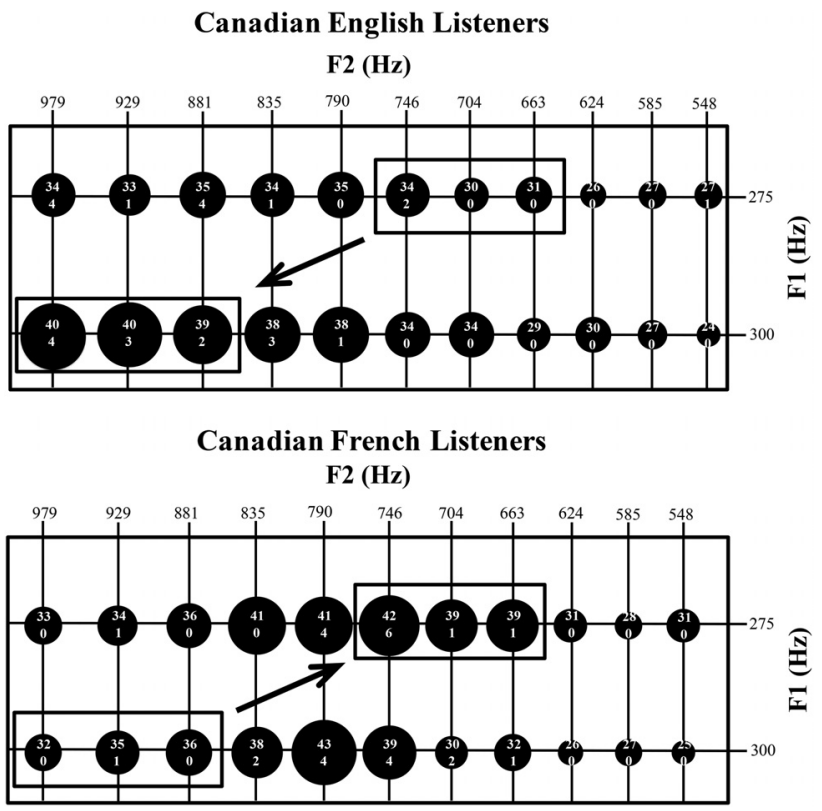

FIG. 2. Composite /u/ (oo) category goodness scores for each vowel in the stimulus array plotted for the English- (top) and French-speaking adults (bottom; experiment 1). The relative differences in the median composite scores (which combines /u/ goodness ratings across all test trials; see text for explanation) are displayed as differences in the size of the corresponding circle (larger for higher median composite score). The upper number within each circle is the median composite score for each stimulus, ranging from 0 (worst)-50 (best). The lower number within each circle is the number of subjects who assigned that token the highest goodness score within the array. The arrows point in the direction that NLM predicts will be easier for each language group to discriminate (experiment 2; see text for explanation). 
the top and the results from the French adults on the bottom. Each circle represents a vowel token. The circle size is scaled to represent the category goodness differences as indexed by the goodness score; the median goodness score for the group is shown in the center of each circle and below it is the number of subjects in the group for whom that token was rated as the best exemplar of the /u/ category (i.e., had the highest goodness score).

Important aspects of the expected language-specific patterns were confirmed. Non-parametric tests showed that there were significant differences in /u/ category goodness scores within each language group. Wilcoxon signed rank tests were conducted to examine the effects of $F 1$ value on goodness scores. This test was implemented within each group, to first compare overall goodness scores for vowels across the two $F 1$ series $(275 \mathrm{~Hz}$ vs $300 \mathrm{~Hz})$ after collapsing across all $F 2$ levels within each series, and then also at each level of $F 2$. For English adults, overall goodness scores were significantly higher $(p<0.01)$ for vowels with higher $F 1$ $(300 \mathrm{~Hz}>275 \mathrm{~Hz})$; this effect was significant for the all vowels with $F 2$ values of $790 \mathrm{~Hz}$ or higher. For French adults there was no overall effect of $F 1$ on category goodness ( $p>0.05$ ); however, when $F 2$ was $548 \mathrm{~Hz}$ category goodness was higher for the token with $F 1$ of $275 \mathrm{~Hz}$.

With respect to $F 2$ frequencies, as shown in Fig. 2, English adults assigned the highest goodness scores to tokens with the highest $F 2$ frequencies, whereas the French adults assigned the highest goodness scores to tokens in the middle of each $F 2$ series. Friedman analysis of variances (ANOVAs) were conducted to analyze rank differences in category goodness related to $F 2$ frequency across subjects. Separate ANOVAs were conducted for each $F 1$ series within each language group. All four ANOVAs revealed highly significant effects of $F 2$ frequency on category goodness. For English adults, $F=52.2(p<0.001)$ for the $F 1=275 \mathrm{~Hz}$ series and $F=83.3(p<0.001)$ for the $F 1=300 \mathrm{~Hz}$ series. For French adults, $F=205.4 .2(p<0.001)$ for the $F 1=275 \mathrm{~Hz}$ series and $F=199.7(p<0.001)$ for the $F 1=300 \mathrm{~Hz}$ series.

To probe the goodness scores in more detail, mean goodness scores were computed for $F 2$ variants grouped into 3 subsets: high $F 2$ tokens $(979,929,881$, and $835 \mathrm{HZ})$, mid $F 2$ tokens $(790,746,704$, and $663 \mathrm{~Hz})$, and low $F 2$ tokens $(625,5895$, and $548 \mathrm{~Hz})$. The subsets scores were computed separately for each $F 1$ series $(F 1=275 \mathrm{~Hz}$ vs $F 1=300 \mathrm{~Hz})$ for each language group. Mean scores on these subsets (high vs mid, mid vs low, and high vs low) were then compared using Wilcoxon sign ranked tests $(p<0.01)$ to evaluate how goodness scores differed as $F 2$ varied. Overall, the results confirmed the language differences shown in Fig. 2. For English adults, goodness scores decreased as $F 2$ decreased; significant differences were found across all 3 comparisons with high $F 2$ subset $>$ mid $F 2$ subset $>$ low $F 2$ subset. This pattern was found for each $F 1$ series. For French adults, the goodness scores were higher for the mid $F 2$ subset compared to both the high $F 2$ and low $F 2$ subsets for the $F 1=275$ series. For the $F 1=300 \mathrm{~Hz}$ series, the goodness scores for the mid and high $F 2$ subsets did not differ and both were higher than the low $F 2$ subset.
Using acoustic and category goodness measures, we then selected two sets of vowels that differ systematically in both their category goodness measures and $F 1$ and $F 2$ formant frequency convergence patterns. These sets are indicated in Figs. 1(c) and 2. The more focal set $\left(u_{4}, u_{5}, u_{6}\right)$ included 3 tokens from the $F 1=275 \mathrm{~Hz}$ series with a relatively small spectral distance between $F 1$ and $F 2$; these tokens were also among those assigned the highest goodness scores by French adults. The less focal set $\left(u_{1}, u_{2}, u_{3}\right)$ included 3 tokens from the $F 1=300 \mathrm{~Hz}$ series in which there is a relatively larger spectral distance between $F 1$ and $F 2$ (compared to the more focal set); these tokens were among those assigned the highest goodness scores by English adults.

Cross-group differences in the category goodness scores assigned to the less focal and more focal vowel sets were evident at the group and individual level. For French adults, median goodness scores were higher for the more focal set ( median $=40)$ than the less focal set (median $=34)$, whereas for English adults the opposite pattern emerged with higher scores for the less focal (median $=39.7$ ) than the more focal set $($ median $=31.7)$. Wilcoxon sign ranks tests confirmed a statistically robust difference in goodness score in the English group (less focal $>$ more focal); the opposite pattern observed in the French group (more $>$ less focal) did not reach statistical significance. ${ }^{3}$ Median tests also revealed significantly higher goodness scores for the French adults compared to the English adults for the more focal set, $\chi^{2}=2.52$, $p<0.05$ ); the reverse pattern (English $>$ French) was observed for the less focal set, but this difference was not statistically supported.

On the individual level, every English subject showed the same pattern with respect to median goodness scores (less focal $>$ more focal), and 9 of the 13 subjects assigned their highest goodness score to a vowel in the less focal set. In the French group, 9 of 13 subjects showed the opposite pattern with respect to median goodness scores (more focal$>$ less focal); 8 of 13 French adults also assigned their highest goodness score to a vowel in the more focal set. However, neither of the later proportions exceed chance predictions.

\section{Discussion}

The results of experiment 1 are clear in showing that Canadian-English and Canadian-French listeners are highly sensitive to the distinct acoustic-phonetic properties that specify their native / $\mathrm{u} /$ category. While listeners from both language groups identified all of the vowel stimuli as unambiguous members of their / $\mathrm{u} /$ category, there was evidence that the differences in their long-term linguistic experiences altered which stimuli within the category were perceived as the best exemplars of $/ \mathrm{u} /$. The best $/ \mathrm{u} /$ exemplars for Canadian-French listeners were more focal with respect to $F 1$ and compared to the best /u/ exemplars for CanadianEnglish listeners.

Additionally, there was a close correspondence between the average formant values reported in previous crosslinguistic English-French vowel production studies and the 
way in which the /u/ category appears to be structured in perception across the two languages. Previous acousticphonetic analyses show that Canadian-French /u/ tends to be produced with significantly lower $F 1$ and $F 2$ values than English /u/ vowels (Escudero and Polka, 2003; MacLeod et al., 2009; Noiray et al., 2011). This pattern is in accord with the present perceptual data, which shows that vowels with lower $F 1$ and $F 2$ values are perceived as better /u/'s by French listeners than English listeners. These findings are not surprising given that these detailed differences must be learned by native speakers, because they have consequences for category boundaries in perception and because they must be accurately reproduced to achieve a native accent.

Finally, and of critical importance to the present study, these cross-language differences in perception provide a stimulus array that can be used to evaluate the rival (although not mutually exclusive) predictions of NLM and NRV. For French, focalization differences and category goodness differences are aligned (i.e., the more focal vowel set contains prototypic French /u/ variants). For English, however, these factors are not aligned (i.e., the less focal vowel set contains prototypic English /u/ variants). Thus, the less-focal/English-prototypic and more-focal/French-prototypic vowel sets define stimulus conditions that we can use to test and compare discrimination performance in English and French listeners in order to disentangle the effects of focalization and prototypicality on directional asymmetries in vowel discrimination.

\section{EXPERIMENT 2: VOWEL DISCRIMINATION}

As described in Sec. I, the NLM model proposes that directional asymmetries reflect language-specific categorization processes, and therefore predicts that listeners should display enhanced sensitivity for discriminating a change from a relatively "poor" to relatively good native vowel category exemplar. On the other hand, the NRV framework proposes that universal perceptual biases and language experience interact to shape vowel perception in mature perceivers, and thus predicts that both factors may contribute to directional asymmetries in adult vowel discrimination. Universal biases are phonetically grounded in formant proximity and drive directional asymmetries in which perceivers show enhanced sensitivity for discriminating a change from a relatively less to a relatively more focal vowel. Experiment 2 was designed to address the merit of each perspective.

Toward this end, we tested whether there are directional asymmetries in Canadian-English and Canadian-French listeners' discrimination of within-category /u/ vowel pairs, using a standard same-different (AX) task. Based on the findings of experiment 1 , we were able to select a set of lessfocal/English-prototypic /u/ vowels and more focal/ Frenchprototypic /u/ vowels, which allowed us to systematically evaluate the impact of these factors. For these select stimuli, NRV and NLM predict asymmetries in the same direction for French adults, but in opposite directions for the English adults. More precisely, if focalization effects alone drive directional asymmetries, then we should observe the same direction effect in both language groups, i.e., enhanced performance when discriminating a change from a relatively less to a relatively more focal / $\mathrm{u} /$ vowel, compared to the reverse direction.

Alternatively, if language-specific categorization processes alone drive direction asymmetries, then we should observe asymmetries going in opposite directions across the two language groups with enhanced discrimination for a change from a relatively poor to a relatively good / $\mathrm{u} /$ vowel exemplar. Importantly, these factors are not mutually exclusive and thus both may play a role. This is the perspective taken in the NRV framework, which proposes that linguistic experience acts to alter initial universal vowel biases. In this case, we hypothesized that we would observe a main effect of focalization, as well as an interaction with native language. Specifically, we predicted that both language groups will show an asymmetry due to focalization, i.e., better discrimination in the less to more focal direction. However, this asymmetry will be more robust for French listeners given that it is aligned with the predicted effects of prototype categorization, and weaker in English perceivers given that it is misaligned with the predicted effects of prototype categorization.

\section{A. Materials and methods}

\section{Subjects}

Thirty adults were recruited in Montreal including 15 monolingual Canadian English speaker-listeners (mean age $=23$ yrs $[\mathrm{SD}=5.1] ; 6$ males) and 15 monolingual Canadian French speaker-listeners (mean age $=36$ yrs $[\mathrm{SD}=12.6]$; 9 males). Subjects were paid for their participation. Eight additional subjects were tested, but excluded from the analysis for the following reasons: equipment failure $(n=1)$; subjects did not follow task instructions $(n=7){ }^{4}$

As in experiment 1, English listeners were from Ontario and French listeners were from Quebec and met the same inclusion criteria as the subjects tested in experiment 1 . Subjects also completed either the Canadian English or Canadian French versions of the LEAP-Q (Marian et al., 2007, which included the self-rating of their speaking and listening proficiency in the other language on the same 10point scale described above in experiment $1[1=$ very poor, $10=$ excellent]). The English subjects' median self-ratings of French proficiency was 2.0 for speaking (range $=1-4$ ) and 2.0 for understanding (range $=1-5$ ). The French subjects' median self-ratings of English proficiency were 5.5 for speaking (range =2-7) and 7.0 for understanding (range $=2-8$ ). Median split tests showed that French subjects rated their English proficiency significantly higher than the English subjects rated their French proficiency for both speaking $(p<0.005)$ and listening $(p<0.005)$. Median split tests also showed no difference between English adult's ratings of French proficiency across experiments 1 and 2 for either speaking or listening. However, French adults rating of English proficiency were significantly higher in experiment 2 than experiment 1 for both skills $(p<0.005)$, despite having applied the same inclusion criteria as experiment 1. 


\section{Stimuli}

A subset of six of the 22 stimuli from experiment 1 were selected for use in this second experiment. The selected stimuli are marked and labeled in Fig. 1(c). As already described, these stimuli were of two types (3 tokens each): Less-focal/English-prototypic /u/ and more-focal/Frenchprototypic /u/.

\section{Procedure and design}

Participants completed an AX discrimination task (e.g., Polka, 1992; Iverson and Kuhl, 1995). On each trial, participants heard two stimuli, and then judged whether they were the "same" or "different." A long inter-stimulus interval (ISI; $1500 \mathrm{~ms}$ ) was used to ensure that a "phonetic level" of analysis was being tapped (i.e., by exerting greater processing demands on auditory working memory and attention; see, e.g., Werker and Logan, 1985; Cowan and Morse, 1986; Repp and Crowder, 1990). Participants initiated a trial by pressing a response key, and then pressed one of two labeled buttons on a keyboard to indicate whether the second stimulus was the same as the first $[\mathrm{A}]$ or different from the first $[\mathrm{X}]$. Prior to the start of the experiment, participants were informed that all of the sound changes that they would hear were subtle, and that they should respond to any differences that they heard in the stimuli. These instructions were meant to replicate the task demands employed in previous studies by Kuhl and her colleagues investigating the effects of category goodness on vowel discrimination in adulthood (Kuhl, 1991; Iverson and Kuhl, 1995, 2000).

Before the test trials started, participants completed six practice trials. During the test trials, participants heard every possible pairing of the six stimuli (including each stimulus being paired with itself), 5 times, in both presentation orders. There were 180 test trials in a session (150 different, 30 [acoustically identical] same). The experiment was broken up into five blocks. Each block had 36 trials, which consisted of one presentation of each possible pairing. No feedback was provided on either the practice or experimental trials. Participants took short breaks as needed.

The experiment was conducted in a sound-treated laboratory room with participants facing a computer screen and with a keyboard in front of them. The stimuli were delivered to both ears through insert earphones (an echo-attenuated plastic tube system terminating in a foam earplug) at $60 \mathrm{~dB}$ sound pressure level, using Presentation (Neurobehavioral Systems, Inc., San Francisco, CA).

\section{B. Results}

Our analysis focused on listeners' discrimination of the nine "cross-set" stimulus pairs that contained a vowel from the less focal set (stimuli $u_{1}, u_{2}, u_{3}$ ) and a vowel from the more focal set (stimuli $u_{4}, u_{5}, u_{6}$ ). To ensure that differences in discrimination performance did not reflect an inherent bias to respond same or different, each subject's performance on each cross-set stimulus pair was converted to an $A^{\prime}$ score. ${ }^{5} A^{\prime}$ is a non-parametric unbiased index of performance that ranges from 0.50 (chance) to 1.0 (perfect discrimination). ${ }^{6}$ For each participant, an $A^{\prime}$ score was computed for each of the nine cross-set stimulus pairs with separate scores computed for each direction of change (less to more focal and more to less focal, e.g., $u_{1} / u_{4}$ and $\left.u_{4} / u_{1}\right)$. The mean $A^{\prime}$ score for each direction of vowel change is plotted for each language group in Fig. 3. These scores were submitted to an ANOVA with order of vowel change (less to more focal vs more to less focal) and stimulus pair $\left(u_{1} / u_{4}\right.$ vs $u_{1} / u_{5}$ vs $u_{1} / u_{6}$ vs $u_{2} / u_{4}$ vs $u_{2} / u_{5}$ vs $u_{2} / u_{6}$ vs $u_{3} / u_{4}$ vs $u_{3} / u_{5}$ vs $\left.u_{3} / u_{6}\right)$ as within-subject factors, and native language (English vs French) as a between-subjects factor. A significant effect of native language $\left[F(1,28)=6.492, p=0.017, \eta^{2} p=0.188\right]$ revealed overall higher sensitivity in French listeners $[M=0.97$, standard error $(\mathrm{SE})=0.005]$ compared to English listeners $(M=0.94, \mathrm{SE}=0.007)$. A robust effect of order of vowel change $\left[F(1,28)=17.098, p=0.000, \eta^{2} p=0.393\right]$ revealed more accurate discrimination in the less to more focal direction $(M=0.97, \mathrm{SE}=0.004)$ compared to the more to less focal direction $(M=0.94, \mathrm{SE}=0.008)$. There was also a significant effect of stimulus pair $[F(8,224)=3.344$, $\left.p=0.001, \eta^{2} p=0.107\right]$ and an order $\mathrm{X}$ stimulus pair interaction $\left[F(8,224)=2.359, p=0.019, \eta^{2} p=0.078\right]$. The latter results show that the direction effect, although observed for each cross-set stimulus pairing, was more robust for some stimulus pairs than others. All other interactions were nonsignificant. $^{7,8}$

Table I shows the mean $A^{\prime}$ scores for each stimulus pairing and order of vowel change collapsed across language groups. To probe the order $\mathrm{X}$ stimulus interaction, simple effects of direction were analyzed via post hoc LSD $t$-tests conducted on each stimulus pairing; the results are reported in Table I. Significant directional asymmetries consistent with a focalization effect emerged for stimulus pairs $u_{1} / u_{4}$, $u_{3} / u_{4}, u_{3} / u_{5}$, and $u_{3} / u_{6}$. The same directional pattern was observed for the remaining stimulus pairs, but did not reach

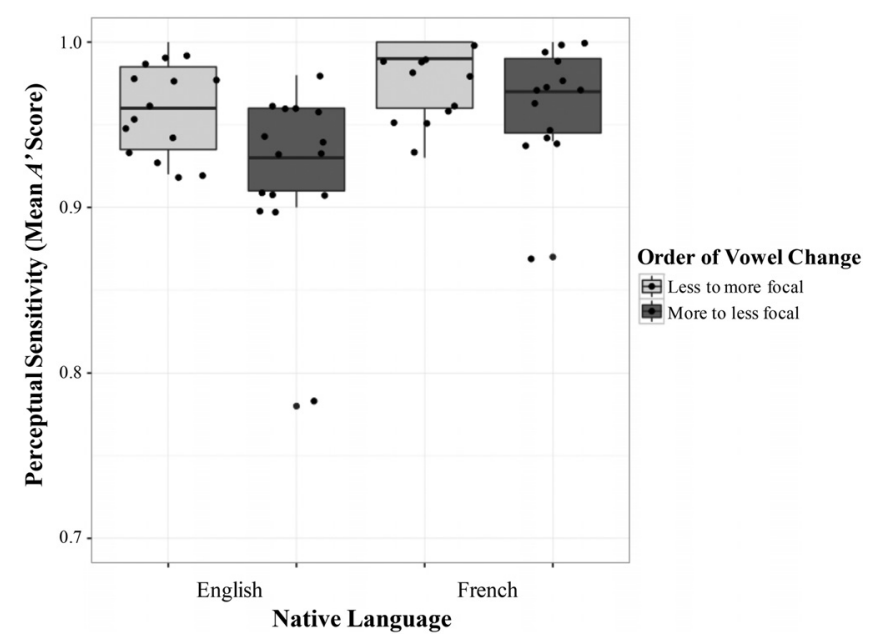

FIG. 3. Native language (English vs French) by order of vowel change (less to more focal vs more to less focal) summary for the cross-set discrimination analysis. Mean percent $A^{\prime}$ scores for all different vowel pairs contrasting a vowel from the less focal vowel set (stimuli $u 1, u 2, u 3$ ) with a vowel from the more focal vowel set (stimuli $u 4, u 5, u 6$ ) for the English and French listeners separately. Error bars represent standard errors. When discrimination performance is at chance, $A^{\prime}=0.5$. 
TABLE I. Mean $A^{\prime}$ values by stimulus pair and presentation order for the cross-set discrimination analyses (statistical values are listed on the far right).

\begin{tabular}{|c|c|c|c|}
\hline \multicolumn{4}{|c|}{ Stimulus Pair Statistical $p$ Values $(\alpha=0.05)$} \\
\hline \multicolumn{4}{|c|}{ Order of Vowel Change } \\
\hline Stimulus pair & Less to more focal & More to less focal & $p$ \\
\hline$u_{1} / u_{4}$ & 0.97 & 0.95 & 0.151 \\
\hline$u_{1} / u_{5}$ & 0.97 & 0.96 & 0.098 \\
\hline$u_{1} / u_{6}$ & 0.97 & 0.96 & 0.095 \\
\hline$u_{2} / u_{4}$ & 0.97 & 0.95 & 0.051 \\
\hline$u_{2} / u_{5}$ & 0.97 & 0.95 & 0.187 \\
\hline$u_{2} / u_{6}$ & 0.97 & 0.96 & 0.114 \\
\hline$u_{3} / u_{4}$ & 0.97 & 0.94 & 0.004 \\
\hline$u_{3} / u_{5}$ & 0.96 & 0.94 & 0.053 \\
\hline$u_{3} / u_{6}$ & 0.97 & 0.91 & 0.000 \\
\hline
\end{tabular}

statistical significance. The direction effect (based on mean $A^{\prime}$ scores for each direction collapsed across stimulus pairs) was also evident in individual performance. Thirteen out of the 15 English participants were better at detecting the less to more focal $/ \mathrm{u} /$ vowel change $(p=0.007$, two-tailed binomial test), and 11 out of the 15 French participants tested were better at detecting the less to more focal /u/ vowel change ( $p=0.118$, two-tailed binomial test). The few French participants who failed to display a direction effect were performing at ceiling. Overall, the results strongly support the predictions of NRV that listeners are universally attuned to formant convergence patterns, and that this bias influences listeners' perception of subtle acoustic differences that fall within the /u/ category.

\section{Discussion}

In experiment 2, we investigated whether directional asymmetries emerged when Canadian-English and Canadian-French listeners discriminated variants that fell within the /u/ category, and, if so, whether those asymmetries were better predicted by focalization, prototypicality, or both factors. Overall, the findings reveal a robust effect of focalization alone. When discriminating pairs of vowels drawn from across the less-focal/English-prototypic and more-focal/French-prototypic sets, both English and French listeners showed the same directional asymmetry-both were better at discriminating the less to more focal $/ \mathrm{u} /$ changes, compared to the reverse direction. This direction effect was more robust for some cross-set stimulus pairs than others. Importantly, this direction effect did not interact with native-language, and thus appears to not be influenced by language experience. These findings provide the first direct evidence of a directional asymmetry in adult vowel discrimination that can be attributed unequivocally to formant focalization. These data provide firm support for a central claim of the NRV framework; namely, that there is a universal perceptual bias favoring vowels with more focal spectral quality that can be observed in adult, as well as infant, listeners.

\section{GENERAL DISCUSSION}

A fundamental goal of research on cross-language speech perception is to explicate the role of both universal and experiential factors (see Cutler, 2012). In the domain of vowel perception, considerable research has focused on examining how listeners' response patterns are influenced by both the intrinsic acoustic-phonetic properties of vowels and phonetic category learning. Research to date has provided ample evidence that the perception of vowels is initially influenced by their relative degree of formant proximity (Polka and Bohn, 2003, 2011), but that this initial vowel bias dynamically adjusts to reflect the structure of the nativelanguage phonological system (Kuhl, 1991; Kuhl et al., 1992; Polka and Werker, 1994; Polka and Bohn, 2011; Pons et al., 2012).

In the current work, we examined further how universal biases and phonetic category learning might interact to shape adults' perception of subtle acoustic-phonetic differences that fall within a given vowel category. Specifically, we investigated the effects of formant proximity and prototypicality on Canadian-English and Canadian-French listeners' perception of a synthetic stimulus array whose members systematically varied in the proximity between their first and second formants. As described in Sec. I, we chose CanadianEnglish and Canadian-French as languages based on certain aspects of their vowel systems. Specifically, the internal structure of their native / $\mathrm{u} /$ categories was expected to differ in ways that would be informative for teasing apart the competing predictions of NRV and NLM concerning asymmetries in within-category vowel discrimination.

In experiment 1, Canadian-English and CanadianFrench listeners' categorization responses to a range of synthetic vowels revealed a perceptual sensitivity to subphonemic properties of native vowel production. Although all members of the stimulus array were consistently identified as /u/ by listeners in both language groups, the best French $/ \mathrm{u} /$ exemplars tended to be more focal compared to the best English /u/ exemplars. This outcome represents a perceptual counterpart to Escudero and Polka's (2003) finding that Canadian-French $/ \mathrm{u} /$ is produced with a greater degree of formant convergence than Canadian-English /u/ (see also MacLeod et al., 2009). In particular, it shows that English and French listeners appear to be especially attuned to the fine-grained acoustic structure of their native $/ \mathrm{u} /$ category.

Experiment 2 then examined the influences of focality and prototypicality on Canadian-English and CanadianFrench listeners' discrimination of a subset of the $/ \mathrm{u} /$ variants (categorized in experiment 1), which systematically differed in both their degree of formant proximity and stimulus goodness ratings, using an AX task. The results were clear in demonstrating that listeners from both language groups showed a directional asymmetry in which their discrimination performance was better when discriminating a change from a less-focal/English-prototypic /u/ to more-focal/ French-prototypic /u/, compared to the reverse. However, there was no evidence that stimulus prototypicality modulated this asymmetry. 
A comparable asymmetry was observed by Schwartz and Escudier (1989) when they examined French adults' discrimination of less versus more focal variants of French /e/. However, interpretation of their data was equivocal due to the absence of data to address the role of prototypicality effects. The current results provide the first direct evidence that directional asymmetries can be modulated by differences in formant convergence exclusively and independently of language-specific prototype categorization. In a recent follow-up study, we replicated the effect of formant proximity in English and French adults using natural speech tokens of French / $\mathrm{u} /$ and English / $\mathrm{u} /$ produced by a bilingual English-French speaker of Canadian English and French (Masapollo et al., 2016). Thus, synthetically controlled stimuli are not required to measure the vowel perception bias documented in the present study.

These findings collectively support one of the basic tenets of the NRV framework; namely, that the perception of vowel sounds is influenced by a universal sensitivity to extreme articulatory postures, which are acoustically specified via formant convergence patterns (Polka and Bohn, 2011; see also Schwartz et al., 2005). The focalization-based perceptual bias documented here also provides critical data in support of the Dispersion-Focalization theory of vowel systems (Schwartz et al., 1997, 2005).

Importantly, although the present findings clearly demonstrate asymmetric patterns that can be attributed exclusively to a universal vowel bias, these findings do not preclude effects of language-specific categorization on directional asymmetries. The NRV framework proposes that both universal and experience-dependent, languagespecific biases play a role in shaping vowel perception, and therefore, that both may contribute to asymmetries. It is possible that directional asymmetries involving the discrimination of relatively large within- and betweencategory differences reveal universal biases that shape the global organization of the vowel space, while asymmetries involving the discrimination of relatively small withincategory differences reveal language-specific biases that shape the local internal structure of native vowel categories. Although the findings from experiment 2 fail to show a modulation of focalization effects by language-specific categorization experience, this may be a limitation of our stimulus array. Specifically, it is possible that prototypicality effects may emerge only during the discrimination of vowel exemplars that fall very close to a prototype stimulus in psychophysical space. In fact, Kuhl's (1991) findings with adults showed larger NLM effects for vowels very close to the prototype stimulus and smaller effects for vowels further from the prototype. Our stimulus array was not designed to assess asymmetries in the narrow region immediately surrounding each native prototype. To accomplish this would require a stimuli set that is carefully constructed to define equivalent and more fine-grained perceptual gradients around the Canadian-English and Canadian-French /u/ prototypes.

The failure to observe effects of prototypically on asymmetries in experiment 2 may be due, at least in part, to slightly weaker differences in category goodness across the less and more focal sets for the French subjects compared to their English peers, which are probably linked to the Canadian-French listeners' overall enhanced sensitivity to the /u/ variants in comparison to their English peers. The later unexpected, but robust finding may be due to differences in the vowel systems of English and French. Canadian-French has a richer inventory of high vowels (/i y $\mathrm{u} /$ ) than English (/i u/). ${ }^{9}$ Thus, this high region of the vowel space is more dense in French than in English, which may explain French listeners' enhanced sensitivity to spectral differences in this part of the vowel space.

An alternative, but not mutually exclusive, explanation for the Canadian-French listeners' enhanced sensitivity to the vowel differences in experiment 2 concerns their exposure/experience with Canadian-English. Even though the French subjects were functionally monolingual, they had undoubtedly still experienced passive exposure to spoken English, and also heard English-accented French, throughout their lives in Quebec. In contrast, the English participants were university students from Ontario studying in Montreal, and therefore, probably had much less passive experience listening to French than the French participants had listening to English. We cannot exclude the possibility that this difference in linguistic experience contributed to the French listeners' perception of the $/ \mathrm{u} /$ variants.

To reduce experience-related differences in overall sensitivity to /u/ vowel differences it would be informative to test Canadian- and/or European-French adults with more limited exposure to English. Additionally, we could examine how Spanish listeners discriminate the /u/ stimuli implemented in the present study. Like English, Spanish only maintains a two-way high vowel contrast $(/ \mathrm{i}-\mathrm{u} /)$, but, like French, the Spanish /u/ is more peripheral (and therefore, more focal between $F 1$ and F2) than English /u/. Accordingly, we would predict that both Spanish listeners and English listeners will show the same focalization-based asymmetry for the cross-set vowel pairs, and a prototypebased asymmetry in opposite directions for English and Spanish perceivers for the within set vowel pairs.

It is also important to recognize that in the broader research context the recent focus on vowel perception asymmetries is not new. Vowel perception asymmetries (referred to as order effects) reported in several earlier studies with adults led Cowan and Morse (1986) to propose the "neutralization hypothesis," which was tested further by Repp and Crowder (1990) and more recently by Karypidis (2007). According to this hypothesis, when listeners hold vowel sounds in memory, the representation of vowel quality drifts toward the neutral vowel [ə]. In this view, vowel order effects can be explained as a memory effect in which some vowel qualities are more subject to decay than others. Overall, this earlier work provided only weak support for the neutralization hypothesis. As well, the AX task implemented in this work often utilized a short ISI which favors auditory (trace) memory coding over phonetic (context coding), or did not use a bias-free measure of discrimination. These task differences make it difficult to interpret many of these earlier findings with respect to the focalization hypothesis. 
The neutralization hypothesis was ultimately considered an inadequate account for vowel order effects by the researchers who proposed it. Nevertheless, their findings and others (e.g., Macmillan et al., 1988) clearly revealed that, along with memory factors, stimulus salience is also a critical factor driving directional asymmetries in vowel perception and other domains as well (e.g., Rosch, 1975). Given that asymmetries provide a way to tag perceptual salience, our research, and research motivated by NLM, has focused on identifying stimulus properties that make some vowels more perceptually salient than others and allow them to serve as anchor or referent points in vowel perception when perceivers are engaged in a phonetic processing task.

According to the NRV framework, it is hypothesized that focalization biases reflect phonetic processes rather than general auditory processes. Thus, we do not expect to observe asymmetries to emerge in all test conditions. However, it is not entirely clear what task demands are needed to elicit a phonetic mode and/or restrict access to auditory memory traces. Several previous studies show that in an AX task, vowel order effects emerge or increase as ISI and stimulus predictability are increased whereas overall performance improves and asymmetries decrease when ISI and stimulus predictability are decreased (e.g., Werker and Logan, 1985; Cowan and Morse, 1986; Repp and Crowder, 1990; Polka and Bohn, 2011). For example, Polka and Bohn (2011) reported a directional asymmetry with a non-native vowel contrast when the ISI was $1500 \mathrm{~ms}$, but not when the ISI was $500 \mathrm{~ms}$. These task factors also likely interact with the magnitude of the stimulus difference presented. Given that overall performance levels were fairly high in experiment 2, we would expect performance to be symmetric and at ceiling if the auditory memory access was enhanced by using a shorter ISI; however, different task effects may emerge for discrimination of more subtle vowel differences. Future research that can clarify the role of both memory aspects and stimulus salience in modulating directional asymmetries linked to focalization and categorization will advance our understanding of the mechanisms involved in vowel processing.

Finally, the present findings have important implications for theoretical models of developmental speech perception, which are centrally concerned with the language-specific reorganization of initial perceptual capacities and biases. While there is no doubt that linguistic experience has a profound and early impact on vowel perception (Kuhl et al., 1992; Polka and Werker, 1994; Polka and Bohn, 2011; Pons et al., 2012), our overall finding that directional asymmetries are driven by a universal sensitivity to formant proximity, independent of language-specific prototype categorization, demonstrates that there are constraints on the extent to which initial vowel biases can be modified through linguistic experience. Recent evidence indicates that this may also be the case in the perception of consonants. Specifically, Nam and Polka (2016) found that young infants from across different linguistic communities are initially biased toward stop consonants over fricative consonants, and that this bias continues to operate within adult language users. At the present time, developmental theories, such as NLM (Kuhl et al.,
2008), have focused almost exclusively on explicating how native-language experience alters speech perception, but such models will ultimately need to provide a more complete account of how universality and language-specificity fit together.

\section{ACKNOWLEDGMENTS}

This research was supported by NSERC Discovery Grant No. 105397 to L.P. and NSERC Discovery Grant No. 312395 to L.M. We are grateful to Anush Nersisyan for assistance with subject recruitment and data-collection, and Vincent Gracco for the generous use of facilities at the Center for Research on Brain, Language, and Music at McGill University. Special thanks to Lauren Franklin for her help with coding in R. This work benefited from helpful discussions with, or comments from, Susan Rvachew, Carol Fowler, Meghan Clayards, Shari Baum, Patricia Kuhl, and James Morgan. Experiment 1 was conducted as part of $\mathbf{M}$. Molnar's doctoral dissertation, and experiment 2 was conducted as part of M. Masapollo's doctoral dissertation.

\footnotetext{
${ }^{1}$ Specific vowels are referred to as focal vowels because they represent maximum formant convergence levels; however, focalization is not all or none, it is a graded effect that gives rise to salience differences across the vowel space. The effects of format convergence are also described in the Quantal Theory of Speech (Stevens, 1989); in this context, the corner vowels $(/ \mathrm{i} /, / \mathrm{a} /, / \mathrm{u} /)$ are called "quantal" vowels.

${ }^{2}$ Note that the English listeners in experiments 1 and 2 were living in Montreal at the time of testing.

${ }^{3}$ This analysis had less power due to one tie in the ranks.

${ }^{4}$ These seven subjects were recruited by an ad in a free newspaper whereas almost all other subjects in both experiments were university students. These subjects did not follow directions and pressed buttons that they were not instructed to use. Their data were removed because we were not confident that they understood or were fully cooperative in the task.

${ }^{5}$ The same results were obtained for all analyses when percent-correct scores were used as the dependent variable.

${ }^{6}$ The following formula was used: $A^{\prime}=0.5+(\mathrm{H}-\mathrm{FA})(1+\mathrm{H}-\mathrm{FA}) /[4 \mathrm{H}$ $(1-\mathrm{FA})]$, where $\mathrm{H}=$ proportion of hits and $\mathrm{FA}=$ proportion of false alarms (Grier, 1971). The false alarm rate was the combined error rate observed on the same trials involving each vowel within the stimulus pair. ${ }^{7} \mathrm{~A}$ robust direction effect was observed when separate ANOVAs were conducted on $A^{\prime}$ scores for each language group.

${ }^{8}$ Reaction times (RTs) collected in experiment 2 also provided converging results. In an ANOVA using RT as the dependent variable, we observed only a significant main effect of order of stimulus presentation $[F(1$, $\left.28)=6.102, p=0.020, \eta^{2} p=0.179\right]$ showing faster response for discriminating less to more focal vowel changes. There was no main effect of native language $[F(1,28)=1.646, p=0.210]$, or native language $\mathrm{X}$ order interaction $[F(1,28)=0.191, p=0.665]$.

${ }^{9}$ In addition, each high vowel in Canadian French has a lax allophonic variant, $[\mathrm{I}],[\mathrm{U}]$ and $[\mathrm{Y}]$. In Canadian English, the tense/lax pairs /i-I/, and /u- $\mathrm{U} /$ occur, but not $/ \mathrm{y}-\mathrm{Y} /$. Further, cross-language vowel production data indicates that the tense/lax high vowel allophones that occur in Canadian French are spectrally closer than the comparable tense/lax vowels that occur Canadian English (Escudero and Polka, 2003).
}

Boë, L.-J. (1999). "Modelling the growth of the vocal tract vowel spaces of newly-born infants and adults: Consequences for ontogenesis and phylogenesis," in Proceedings of the International Congress of Phonetic Sciences, pp. 2501-2504.

Boersma, P., and Weenink, D. (2016). "Praat: Doing phonetics by computer" [Computer program]. Version 6.0.19, http://www.praat.org/ (Last viewed October 15, 2015).

Bothorel, A., Simon, P., Wioland, F., and Zerling, J. P. (1986). Cinéradiographie des Voyelles et Consonnes du Français (Cineradiographic Study of French Vowels and Consonants) (Institut de Phonétique de Strasbourg, Strasbourg, France), pp. 1-296. 
Cowan, N., and Morse, P. (1986). "The use of auditory and phonetic memory in vowel discrimination,” J. Acoust. Soc. Am. 79, 500-507.

Cutler, A. (2012). Native Listening: Language Experiences and the Recognition of Spoken Words (MIT Press, Cambridge, MA), pp. 1-576.

Diesch, E., Iverson, P., Kettermann, A., and Siebert, C. (1999). "Measuring the perceptual magnet effect in the perception of /i/ by German listeners," Psych. Res. 62, 1-19.

Dufour, S., Brunelliere, A., and Nguyen, N. (2013). "To what extent do we hear phonemic contrasts in a non-native regional variety? Tracking the dynamics of perceptual processing with EEG," J. Psycholing. Res. 42, 161-173.

Escudero, P., and Polka, L. (2003). "A cross-language study of vowel categorization and vowel acoustics," in Proceedings of the International Congress of Phonetic Sciences, edited by M. J. Sole, D. Recansens, and J. Romero, Barcelona, Spain, pp. 861-864.

Feldman, N., Griffiths, T. L., and Morgan, J. (2009). "The influences of categories on perception: Explaining the perceptual magnet effect as optimal statistical inference," Psychol. Rev. 116(4), 752-782.

Frieda, E. M., Walley, A. C., Flege, E. J., and Sloane, M. E. (1999). "Adults' perception of native and nonnative vowels: Implications for the perceptual magnet effect," Percept. Psychophys. 61(3), 561-577.

Grier, J. B. (1971). "Nonparametric indexes for sensitivity and bias: Computing formulas," Psychol. Bull. 75, 424-429.

Iverson, P., and Kuhl, P. K. (1995). "Mapping the perceptual magnet effect for speech using signal detection theory and multidimensional scaling," J. Acoust. Soc. Am. 97, 553-562.

Iverson, P., and Kuhl, P. K. (2000). "Perceptual magnet and phoneme boundary effects in speech perception: Do they arise from a common mechanism?," Percept. Psychophys. 62, 874-886.

Johnson, K., Flemming, E., and Wright, R. (1993). "The hyperspace effect: Phonetic targets are hyperarticulated," Language 69, 505-528.

Karypidis, C. (2007). "Order effects and vowel decay in short-term memory: The neutralization hypothesis," in Proceedings of the International Congress of Phonetic Sciences, Saarbrücken, pp. 657-660.

Kent, R. D., and Read, C. (2002). The Acoustic Analysis of Speech (Singular/Thomson Learning, San Diego, CA), pp. 1-320.

Kuhl, P. K. (1991). "Human adults and human infants show a 'Perceptual Magnet Effect' for the prototypes of speech categories: Monkeys do not," Percept. Psychophys. 50, 93-107.

Kuhl, P. K. (1993). "Innate predispositions and the effects of experience in speech perception: The native language magnet theory," in Developmental Neurocognition: Speech and Face Processing in the First Year of Life, edited by B. deBoysson-Bardies, S. de Schonen, P. Jusczyk, P. McNeilage, and J. Morton (Kluwer Academic Publishers, Dordrecht, Netherlands), pp. 259-274.

Kuhl, P. K., Conboy, B. T., Coffey-Corina, S., Padden, D., Rivera-Gaxiola, M., and Nelson, T. (2008). "Phonetic learning as a pathway to language: New data and native language magnet theory expanded (NLM-e)," Philos. Trans. Royal Soc. B 363, 979-1000.

Kuhl, P. K., Williams, K. A., Lacerda, F., Stevens, K. N., and Lindblom, B. (1992). "Linguistic experience alters phonetic perception in infants by 6 months of age," Science 255, 606-608.

Lively, S. E., and Pisoni, D. B. (1997). "On prototypes and phonetic categories: A critical assessment of the perceptual magnet effect in speech perception,” J. Exp. Psych. Human Percept. Perf. 23(6), 1665-1679.

Lotto, A. J., Kluender, K. R., and Holt, L. L. (1998). "Depolarizing the perceptual magnet effect," J. Acoust. Soc. Am. 103(6), 3648-3655.

MacLeod, A., Stoel-Gammon, C., and Wassink, A. B. (2009). "Production of high vowels in Canadian English and Canadian French: A comparison of early bilingual and monolingual speakers," J. Phonetics 37(4), 374-387.

MacMillan, N. A., Goldberg, R. F., and Braida, L. D. (1988). "Resolution for speech sounds: Basic sensitivity and context memory on vowel and consonant continua," J. Acoust. Soc. Am. 84(4), 1262-1280.

Maeda, S. (1979). "An articulatory model of the tongue based on a statistical analysis," J. Acoust. Soc. Am. 65, S22.

Maeda, S. (1990). "Compensatory articulation during speech: Evidence from the analysis and synthesis of vocal tract shapes using an articulatory model," in Speech Production and Speech Modeling, edited by W. L.
Hardcastle and A. Marshal (Kluwer Academic, Dordrecht, The Netherlands), pp. 131-149.

Marian, V., Blumfeld, H. K., and Kaushanskaya, M. (2007). "Language Experience and Proficiency Questionnaire (LEAP-Q)," J. Speech, Lang. Hear. Res. 50(4), 940-967 (2007).

Martin, P. (2002). "Vowel system of Quebec French. From acoustics to phonology," La Linguistique 38, 71-88.

Masapollo, M., Polka, L., and Ménard, L. (2016). "The role of visualphonetic information from lip movements on the natural referent vowel bias," Poster presented at the 15th Meeting of the Association for Laboratory Phonology, Cornell University, Ithaca, NY.

Ménard, L., Davis, B., Boë, L.-J., and Roy, J.-P. (2009). "Producing American-English during vocal tract growth: A perceptual categorization study of synthesized vowels," J. Speech, Lang., Hear. Res. 52, 1268-1285.

Ménard, L., Schwartz, J.-L., and Boë, L.-J. (2004). "The role of vocal tract morphology in speech development: Perceptual targets and sensori-motor maps for French synthesized vowels from birth to adulthood," J. Speech, Lang. Hear. Res. 47, 1059-1080.

Nam, Y.-J., and Polka, L. (2016). "The phonetic landscape in infant consonant perception is an uneven terrain," Cognition 155, 57-66.

Noiray, A., Cathiard, M.-A., Ménard, L., and Abry, C. (2011). "Test of the movement expansion model: Anticipatory vowel lip protrusion and constriction in French and English speakers," J. Acoust. Soc. Am. 129(1), 340-349.

Peterson, G. E., and Barney, H. L. (1952). "Control methods used in a study of the vowels," J. Acoust. Soc. Am. 24, 175-184.

Polka, L. (1992). "Characterizing the influence of native language experience on adult speech perception," Percept. Psychophys. 52(1), 37-52.

Polka, L., and Bohn, O. (1996). "A cross-language comparison of vowel perception in English-learning and German-learning infants," J. Acoust. Soc. Am. 100, 577-592.

Polka, L., and Bohn, O. (2003). "Asymmetries in vowel perception," Speech Commun. 41, 221-231.

Polka, L., and Bohn, O. (2011). "Natural Referent Vowel (NRV) framework: An emerging view of early phonetic development," J. Phonetics 39, 467-478.

Polka, L., and Werker, J. F. (1994). "Developmental changes in perception of non-native vowel contrasts," J. Exp. Psych. Human Percept. Perf. 20(2), 421-435 (1994).

Pons, F., Albareda-Castellot, B., and Sebastian-Gallés, N. (2012). "The interplay between input and initial biases: Asymmetries in vowel perception during the first year of life," Child Develop. 83(3), 965-976.

Repp, B. H., and Crowder, R. G. (1990). "Stimulus order effects in vowel discrimination,” J. Acoust. Soc. Am. 88, 2080-2090.

Repp, B. H., Healy, A. F., and Crowder, R. G. (1979). "Categories and context in the perception of isolated steady-state vowels," J. Exp. Psychol.: Human Percept. Perform. 5, 129-145.

Rosch, E. (1975). "Cognitive reference points," Cognition 7, 532-547.

Schwartz, J.-L., Abry, C., Boë, L.-J., Ménard, L., and Vallée, N. (2005). "Asymmetries in vowel perception, in the context of the DispersionFocalization Theory," Speech Commun. 45, 425-434.

Schwartz, J.-L., Boë, L.-J., Vallée, N., and Abry, C. (1997). "The Dispersion-Focalization Theory of vowel systems," J. Phonetics 25, 255-286.

Schwartz, J.-L., and Escudier, P. (1989). "A strong evidence for the existence of a large-scale integrated spectral representation in vowel perception," Speech Commun. 8, 235-259.

Stevens, K. N. (1989). "On the quantal nature of speech," J. Phonetics 17, $3-46$.

Tyler, M. D., Best, C. T., Faber, A., and Levitt, A. G. (2014). "Perceptual assimilation and discrimination of non-native vowel contrasts," Phonetica 71, 4-21.

Werker, J. F., and Logan, J. S. (1985). "Cross-language evidence for three factors in speech perception," Percept. Psychophys. 37, 35-44.

Whalen, D. H., Magen, H. S., Pouplier, M., Kang, A. M., and Iskarous, K. (2004). "Vowel production and perception: Hyperarticulation without a hyperspace effect," Lang. Speech 47, 155-174.

Zwicker, E., and Terhardt, E. (1980). "Analytical expressions for criticalband rate and critical bandwidth as a function of frequency," J. Acoust. Soc. Am. 68, 1523-1525. 